\title{
Small Molecule Screening Shared Resource
}

National Cancer Institute

\section{Source}

National Cancer Institute. Small Molecule Screening Shared Resource. NCI Thesaurus.

Code C39516.

The Small Molecule Screening Shared Resource is dedicated to provide Cancer Center members with access to high throughput screening of structurally-diverse, drug-like chemicals in appropriate biological assays. The resource provides screening and liquid handling equipment, informatics for data handling and analysis, assay design expertise, centralized storage of compound libraries and develops new methodologies for screening of small molecule agents that inhibit cancer phenotypes in cellular or animal models. 